\title{
ROOT SYSTEM OF SINGULAR PERTURBATIONS OF THE HARMONIC OSCILLATOR TYPE OPERATORS
}

\author{
BORIS MITYAGIN AND PETR SIEGL
}

\begin{abstract}
We analyze perturbations of the harmonic oscillator type operators in a Hilbert space $\mathcal{H}$, i.e. of the self-adjoint operator with simple positive eigenvalues $\mu_{k}$ satisfying $\mu_{k+1}-\mu_{k} \geq \Delta>0$. Perturbations are considered in the sense of quadratic forms. Under a local subordination assumption, the eigenvalues of the perturbed operator become eventually simple and the root system contains a Riesz basis.
\end{abstract}

\section{INTRODUCTION}

This paper deals with the spectrum and eigensystem of perturbations of a selfadjoint operator $A$ in a Hilbert space $\mathcal{H}$. The operator $A$ is of the one dimensional harmonic oscillator type, i.e. its eigenvalues are simple, positive and satisfy

$$
A \psi_{n}=\mu_{n} \psi_{n}, \quad\left\|\psi_{n}\right\|=1, \quad \mu_{1}>0, \quad \exists \Delta>0, \forall n \in \mathbb{N}, \mu_{n+1}-\mu_{n} \geq \Delta ;
$$

see also Remark 1. The perturbations are not assumed to be symmetric, therefore the studied operator $T$ is generically non-self-adjoint (and non-normal), hence the spectrum typically does not remain real and the basis property of eigensystem is no longer guaranteed.

The main aim is to extend (to cover in particular the $\delta$ potential) the results of 3, 4, 5] on sufficient conditions on perturbations guaranteeing that the eigensystem of the perturbed operator contains a Riesz basis. Problems of this type are studied in many works, both classical ones as [10, 19, 20, and more recent ones, for instance, [5, 26, 32, 33, 35].

The essential issue in the analysis is that the gaps between the eigenvalues of the unperturbed operator $A$ do not grow. Assuming that the gaps grow, i.e. $\mu_{n+1}-$ $\mu_{n} \rightarrow+\infty$ and the perturbation $B$ is bounded, Kato proved, $c f$. 19, Thm. V.4.15a, Lem. V.4.17a], that the system of eigenfunctions of $A+B$, plus possibly finite number of associated functions, contains a Riesz basis. The analogous classical theorem allowing also unbounded perturbations can be found in [10, Thm. XIX.2.7]; nevertheless, the growth condition of the gaps is preserved. Constant gaps are allowed in 5; however, only bounded perturbations satisfy the assumption in 5] and the result is weaker, since only the Riesz basis with brackets is claimed.

Adduci and Mityagin overcome the problem of the non-growing gaps in the study of the harmonic oscillator, $c f$. [3] , by

a) using the Hilbert transform as the important technical tool,

Date: 28th September 2015.

2010 Mathematics Subject Classification. 47A55, 47A70, 34L10.

Key words and phrases. non-self-adjoint operators, harmonic oscillator, Riesz basis, quadratic forms, singular potentials.

P.S. appreciates the kind hospitality and support of OSU allowing his stays there in November 2012 and July 2013 and acknowledges the SCIEX Program; the work has been conducted within the SCIEX-NMS Fellowship, project 11.263. Till March 2013, P.S. has been supported by a grant within the scope of FCT's project PTDC/ MAT/ 101007/2008 and partially by FCT's projects PTDC/ MAT/ 101007/2008 and PEst-OE/MAT/UI0208/2011. 
b) replacing the condition of the boundedness of perturbation $B$ by

$$
\left\|B \psi_{n}\right\| \rightarrow 0 \text { as } n \rightarrow \infty
$$

c) using the following result of Kato;

Criterion 1 ([19, Lem.V.4.17a]). Let $\left\{P_{j}\right\}_{j=0}^{\infty}$ be a complete family of orthogonal projections in a Hilbert space $\mathcal{H}$, and let $\left\{Q_{j}\right\}_{j=0}^{\infty}$ be a family of (not necessarily orthogonal) projections such that $Q_{j} Q_{k}=\delta_{j k} Q_{j}$. Assume that

$$
\begin{aligned}
& \operatorname{Rank} P_{0}=\operatorname{Rank} Q_{0}<\infty, \\
& \forall f \in \mathcal{H}, \quad \sum_{j=1}^{\infty}\left\|P_{j}\left(Q_{j}-P_{j}\right) f\right\|^{2} \leq c_{0}\|f\|^{2},
\end{aligned}
$$

where $c_{0}$ is a constant smaller than 1 . Then there is a $W \in \mathscr{B}(\mathcal{H})$ with $W^{-1} \in$ $\mathscr{B}(\mathcal{H})$ such that $Q_{j}=W^{-1} P_{j} W$ for $j \in \mathbb{N}_{0}:=\mathbb{N} \cup\{0\}$.

The condition (2) has been called by Shkalikov a local subordinate condition, see the discussion in [27] and also [4, Sec.1] for some explanations on this wording. Criterion 1 substitutes for the often used Bari-Markus criterion, which is given with more restrictive conditions

$$
\begin{aligned}
& \sum_{j=0}^{\infty}\left\|Q_{j}-P_{j}\right\|^{2}<\infty \\
& \operatorname{Rank} P_{j}=\operatorname{Rank} Q_{j}<\infty, \quad j \in \mathbb{N}_{0},
\end{aligned}
$$

see e.g. [15, Chap.6, Sec. 5.3, Thm. 5.2] or [20].

For the harmonic oscillator in $L^{2}(\mathbb{R})$, the property of Hermite functions,

$$
\max _{x \in \mathbb{R}}\left|h_{n}(x)\right| \leq C n^{-\frac{1}{12}}, \quad n \in \mathbb{N},
$$

can be used to show that (2) is satisfied for $B$ being, for instance, a multiplication operator by $V \in L^{2}(\mathbb{R})$ what is consistently used in 3 .

The results of [3] for the harmonic oscillator have been extended in [4] to the abstract setting with the possibility of the controlled condensation of eigenvalues, i.e. $\mu_{n+1}-\mu_{n} \geq \kappa n^{\omega-1}$ with fixed $\kappa>0$ and $\omega>1 / 2$, or the finite clustering of eigenvalues, i.e. there exist fixed values $q>0$ and $\delta>0$ such that $\mu_{n+q}-\mu_{n} \geq \delta$ for all $n$. In the latter case, similar results have been obtained in [26] using different methods. However, the $\delta$ potential is not covered by the assumption (2) which is essential in [3, 4, 26].

In this paper, we consider perturbations of $A$ in the sense of quadratic forms. (Such a setting has been considered in [5] under the form $p$-subordination assumption, $c f$. (5) below.) At first we define the quadratic form $t:=a+b$, where $a$ corresponds to $A$ and $b$ is the perturbation. The perturbed operator $T$ is associated with the form $t$, see Section 2 for details. Such a framework is one way how to include singular perturbations, $c f$. [19, Chap.VI.3.-4.] or [29, §.1.] in self-adjoint setting. Our main example is the harmonic oscillator in $L^{2}(\mathbb{R})$ perturbed by the $\delta$ potential with complex coupling. We remark that the form $b$ does not need to be closed and therefore it does not need to represent an operator in a considered Hilbert space $\mathcal{H}$, distributional potentials are typical cases.

A straightforward reformulation of the condition (2), coming from [3, 4, 26], would be

$$
\left\|B \psi_{n}\right\|^{2}=\sum_{m=1}^{\infty}\left|b\left(\psi_{n}, \psi_{m}\right)\right|^{2} \rightarrow 0 \text { as } n \rightarrow \infty .
$$


Nevertheless, the analysis of the harmonic oscillator perturbed by the $\delta$ potential, i.e. $b(\phi, \psi)=\phi(0) \overline{\psi(0)}$, reveals that the condition (11) is not satisfied, $c f$. (19)-(20) in Section 5 ,

Our results are obtained under the assumption

$$
\exists \alpha>0, \quad \exists M_{b}>0, \quad \forall m, n \in \mathbb{N}, \quad\left|b\left(\psi_{m}, \psi_{n}\right)\right| \leq \frac{M_{b}}{m^{\alpha} n^{\alpha}},
$$

see also Remark 4 at the end of Section 4. This extends the previously considered classes of perturbations. For the harmonic oscillator particularly, it means a step towards singular potentials including the mentioned $\delta$. This paper yields also a partially new version of the proof of the main result in 3 for some cases. More precisely, unlike in 3, 4, where the important technical tool was the Hilbert transform, only the Schur test is used here. The connection to the previous work 3 is explained in Section 5.2. Moreover, the result of [3] is extended to perturbations by potentials $V \in L(p, \tau), 1 \leq p<\infty, \tau / 4+t(2 p)<0$, cf. Theorem 7 and (23), (25) in Section 5.2, in particular we obtain the extension to potentials $V \in L^{p}(\mathbb{R})$, $1 \leq p<2$. The overall result on perturbations of the harmonic oscillator is formulated in Corollary 8 in Section 5.3 . Further detailed analysis of the spectrum of a harmonic oscillator perturbed by point interactions can be found in 22, 23.

This paper as well as mentioned previous works aim to find sufficient conditions for the Riesz basisness of the eigensystem. However, the negative results, i.e. the fact that the eigensystem is not a Riesz basis (or even a basis), have been obtained particularly for complex oscillators in [7, 8, 9, 17, 18, 28, and just recently in [24.

The paper is organized as follows. In Section 2, we define the operator $T$ and recall some known facts. The main results on the localization of the spectrum and Riesz basisness of the eigensystem are contained and proven in Section 3 . In Section 4, we collect technical lemmas used in the proofs of main results. Section 5 consists of examples and the result for the harmonic oscillator. Conclusions and short discussion are contained in the final Section 6 .

\section{DEFINITION OF THE OPERATOR AND PRELIMINARIES}

The definition of the operator $T$ is based on the first representation theorem [19, Thm.VI.2.1] that provides the unique correspondence between the $m$-sectorial operator $T$ and the closed sectorial form $t$. The detailed definition of the operator $T$ can be also found in [5, Sec.2.].

The self-adjoint operator $A$ is associated, via the second representation theorem [19. Thm.VI.2.23], with a quadratic form

$$
a(\psi, \psi):=\left\|A^{\frac{1}{2}} \psi\right\|^{2}, \quad \operatorname{Dom}(a):=\operatorname{Dom}\left(A^{\frac{1}{2}}\right) .
$$

We consider perturbations by a form $b$ satisfying the condition (4). It follows that $b$ is a form $p$-subordinated perturbation of $a$, i.e. there exist $0 \leq p<1$ and $C>0$ such that

$$
\forall f \in \operatorname{Dom}(a), \quad|b(f, f)| \leq C(a(f, f))^{p}\|f\|^{2(1-p)},
$$

see Lemma 4. The form $p$-subordination implies the form relative boundedness of $b$ with respect to $a$ with the bound 0 . The perturbed operator $T$ is defined as the operator associated, via the first representation theorem [19, Thm.VI.2.1], with a sectorial form

$$
t:=a+b, \quad \operatorname{Dom}(t)=\operatorname{Dom}(a) .
$$

The domains of $t$ and $a$ are the same due to the $p$-subordination, nevertheless, $\operatorname{Dom}(T)$ and $\operatorname{Dom}(A)$ are typically different. The form relative boundedness with the bound 0 together with [19, Thm.VI.3.4] imply that $T$ has a compact resolvent. 
The definition of $T$ can be also reformulated as

$$
T=A^{\frac{1}{2}}(I-B(0)) A^{\frac{1}{2}} .
$$

Here $B(z), z \in \mathbb{C}$, is the operator uniquely determined by the bounded form $b((z-$ $\left.A)^{-1 / 2} \cdot(\bar{z}-A)^{-1 / 2} \cdot\right)$, i.e. $\langle B(z) f, g\rangle=b\left((z-A)^{-1 / 2} f,(\bar{z}-A)^{-1 / 2} g\right)$ for all $f, g \in \mathcal{H}$. The square root of $z-A$ is defined as

$$
(z-A)^{-\frac{1}{2}} f:=\sum_{k \in \mathbb{N}}\left(z-\mu_{k}\right)^{-\frac{1}{2}} c_{k} \psi_{k},
$$

for $f=\sum_{k \in \mathbb{N}} c_{k} \psi_{k}$ and $w^{s}:=|w|^{s} e^{\mathrm{i} s \arg w},-\pi<\arg w \leq \pi$, for $0 \neq w \in \mathbb{C}$ and $s \in \mathbb{R}$. For all $z \in \rho(A)$,

$$
z-T=(z-A)^{\frac{1}{2}}(I-B(z))(z-A)^{\frac{1}{2}} .
$$

This relation yields a suitable representation of the resolvent of $T$, i.e.

$$
(z-T)^{-1}=(z-A)^{-\frac{1}{2}}(I-B(z))^{-1}(z-A)^{-\frac{1}{2}}
$$

provided $I-B(z)$ is invertible and $z \in \rho(A)$. Formulas of this type are also derived in [5, Lem.1.], [19, Chap.VI.3.1.].

Remark 1 . We have started with the operator $A$ with eigenvalues satisfying $\mu_{n+1}-$ $\mu_{n} \geq \Delta, \Delta>0$. However, to simplify all formulas, we will assume that $\Delta=1$ and $\mu_{1} \geq 1$ in the sequel. (Without loss of generality this can be always achieved by considering $\Delta^{-1}(A+c I)$ with a suitably chosen $c \in \mathbb{R}_{+}$.) Eigenvalues $\mu$ then satisfy

$$
\mu_{k} \geq k
$$

\section{Main Results}

Set

$$
\begin{aligned}
\Pi_{0} & :=\{z \in \mathbb{C}:-h<\operatorname{Re} z<(N+3 / 2),|\operatorname{Im} z|<h\} \\
\Pi_{k} & :=\left\{z \in \mathbb{C}:\left|z-\mu_{k}\right|<1 / 2\right\}, \quad \Gamma_{k}:=\partial \Pi_{k}, \\
\Pi & :=\Pi_{0} \cup\left(\bigcup_{j>N+1} \Pi_{j}\right),
\end{aligned}
$$

where $N \in \mathbb{N}$ and $h>1$ are determined in the following way. The aim is to localize the spectrum of $T$. We will succeed if we guarantee that $\|B(z)\| \leq 1 / 2$ for $z$ outside of $\Pi$.

Let $N \equiv N(\alpha)$ be an integer such that

$$
\forall n>N, \quad M_{b} C(2 \alpha) \sigma_{2 \alpha}(n) \leq \frac{1}{2},
$$

where $M_{b}$ is as in (44) and $C(\alpha), \sigma_{\alpha}(n)$ are introduced in Lemma 5 below. $h>1$ is selected such that

$$
2 M_{b}\left(\frac{1}{h} \sum_{k=1}^{N+2} \frac{1}{k^{2 \alpha}}+D(2 \alpha) \tau_{2 \alpha}(h)\right) \leq \frac{1}{2},
$$

where $D(\alpha), \tau_{\alpha}(h)$ are introduced in Lemma 6 .

Proposition 2. Let conditions (1), (4) hold and let $N$ and $h$ satisfy the conditions (9) and (10), respectively. Then the eigenvalues of $T$ are contained in the interior of $\Pi$, cf. (8). Moreover, Riesz projections

$$
\begin{aligned}
S_{N+1} & :=\frac{1}{2 \pi \mathrm{i}} \int_{\Gamma_{0}}(z-T)^{-1} \mathrm{~d} z, \\
P_{j} & :=\frac{1}{2 \pi \mathrm{i}} \int_{\Gamma_{j}}(z-T)^{-1} \mathrm{~d} z \text { for } j>N+1,
\end{aligned}
$$


are well-defined and

$$
\operatorname{Rank} S_{N+1}=N+1, \quad \operatorname{Rank} P_{j}=1 \text { for } j>N+1 .
$$

Proof. At first we show that $(z-T)^{-1}$ is bounded for every $z \notin \Pi_{0} \cup_{j>N+1} \Pi_{j}$. Using the resolvent factorization (6), it suffices to prove that $\|B(z)\| \leq 1 / 2$. Let $f=\sum_{j=1}^{\infty} f_{j} \psi_{j} \in \mathcal{H}$, then

$$
\begin{aligned}
\|B(z) f\|^{2} & =\sum_{k=1}^{\infty}\left|\left\langle B(z) f, \psi_{k}\right\rangle\right|^{2}=\sum_{k=1}^{\infty}\left|\sum_{j=1}^{\infty} \frac{f_{j} b\left(\psi_{j}, \psi_{k}\right)}{\left(z-\mu_{j}\right)^{\frac{1}{2}}\left(z-\mu_{k}\right)^{\frac{1}{2}}}\right|^{2} \\
& \leq M_{b}^{2} \sum_{k=1}^{\infty} \frac{1}{k^{2 \alpha}\left|\mu_{k}-z\right|}\left(\sum_{j=1}^{\infty} \frac{\left|f_{j}\right|}{j^{\alpha}\left|\mu_{j}-z\right|^{\frac{1}{2}}}\right)^{2} \\
& \leq M_{b}^{2}\left(\sum_{k=1}^{\infty} \frac{1}{k^{2 \alpha}\left|\mu_{k}-z\right|}\right)^{2}\|f\|^{2} .
\end{aligned}
$$

Let $\operatorname{Re} z \in\left[\mu_{n}-1 / 2, \mu_{n+1}-1 / 2\right]$ and $z \notin \Pi_{n}, n \geq N$. We apply inequalities from Lemma 5 and we obtain

$$
\|B(z)\| \leq M_{b} C(2 \alpha) \sigma_{2 \alpha}(n) \leq \frac{1}{2}
$$

for $n>N$, where $N$ is chosen above, $c f$. (9).

The next step is estimate outside of $\Pi_{0}$. If $\operatorname{Re} z=-h$, then

$$
\|B(z)\| \leq M_{b} \sum_{k=1}^{\infty} \frac{1}{k^{2 \alpha}(k+h)} \leq M_{b} D(2 \alpha) \tau_{2 \alpha}(h) \leq \frac{1}{2},
$$

for $h$ selected as above, $c f$. (10).

If $\operatorname{Re} z \in[-h,(N+3 / 2)]$, then $|\operatorname{Im} z| \geq h$. We denote $z=s+\mathrm{i} h$, then

$$
\begin{aligned}
\|B(z)\| & \leq M_{b} \sum_{k=1}^{\infty} \frac{1}{k^{2 \alpha} \sqrt{\left(\mu_{k}-s\right)^{2}+h^{2}}} \leq 2 M_{b} \sum_{k=1}^{\infty} \frac{1}{k^{2 \alpha}\left(\left|\mu_{k}-s\right|+h\right)} \\
& \leq 2 M_{b}\left(\sum_{k=1}^{N+2} \frac{1}{h k^{2 \alpha}}+\sum_{k=N+3}^{\infty} \frac{1}{k^{2 \alpha}(k-N-2+h)}\right) \\
& \leq 2 M_{b}\left(\frac{1}{h} \sum_{k=1}^{N+2} \frac{1}{k^{2 \alpha}}+D(2 \alpha) \tau_{2 \alpha}(h)\right) \leq \frac{1}{2}
\end{aligned}
$$

where we use (7), (10), and inequalities

$$
(a+b) / 2 \leq \sqrt{a^{2}+b^{2}}, \quad-h \leq s \leq N+3 / 2 .
$$

The standard argument, based on [11, Lem.VII.6.7], shows that

$$
\text { Trace } \frac{1}{2 \pi \mathrm{i}} \int_{\Gamma_{n}}(z-A)^{-\frac{1}{2}}(I-t B(z))^{-1}(z-A)^{-\frac{1}{2}} \mathrm{~d} z, 0 \leq t \leq 1,
$$

is a continuous integer valued function. Therefore it is constant and the second part of the claim follows.

The obvious corollary is that the eigenvalues $\lambda_{n}$ of $T$ become eventually simple (for $n>N+1$ ) and localized around those of the unperturbed operator $A$, while the first part of the spectrum is localized in $\Pi_{0}$; it is important that there is only a finite number of eigenvalues in $\Pi_{0}$. The latter also means that the eigensystem of $T$ contains at most a finite number of root vectors associated with different eigenvalues $\left\{\lambda_{n}\right\}_{n=1}^{N_{0}}, N_{0} \leq N+1$, in $\Pi_{0}$ with algebraic multiplicities $\left\{m_{n}\right\}_{n=1}^{N_{0}}$, 
$\sum_{n=1}^{N_{0}} m_{n}=N+1$, and the rest, $\left\{\lambda_{n}\right\}_{n>N+1}$, consists of eigenvectors $\left\{\phi_{n}\right\}$ related to eigenvalues in $\cup_{j>N} \Pi_{j}$ of both algebraic and geometric multiplicity one.

Remark 2. Proposition 2 serves to define the Riesz projections $S_{N+1}$ and $P_{j}$ that are further analyzed in Theorem 3 . If we wish to localize the eigenvalues of $T$ more precisely, we can modify $\Pi_{k}, k>N$, to be circles with radii $r_{k} \rightarrow 0$ instead of $1 / 2$. Then the straightforward modification of estimates (12), (13), and Lemma 5 yields that $r_{k}$ does decay as $\mathcal{O}\left(k^{-2 \alpha}\right)$.

A detailed eigenvalue asymptotics of a harmonic oscillator perturbed by point interactions, see Section 5 below, can be found in [22, 23.

To formulate the main result we denote $\left\{P_{n}^{0}\right\}$ the one dimensional spectral projections of $A$ related to eigenvalues $\left\{\mu_{n}\right\}$, i.e.

$$
P_{n}^{0}:=\frac{1}{2 \pi \mathrm{i}} \int_{\left|z-\mu_{n}\right|=\frac{\Delta}{2}}(z-A)^{-1} \mathrm{~d} z, \quad n \in \mathbb{N} .
$$

Theorem 3. Let conditions (11) and (4) hold. Then there exists a bounded operator $W$ with bounded inverse such that projectors $\left\{P_{n}\right\}$ and $S_{N+1}$, cf. (11), satisfy

$$
W P_{n} W^{-1}=P_{n}^{0}
$$

for all $n>N+1$ and

$$
W S_{N+1} W^{-1}=\sum_{n=1}^{N+1} P_{n}^{0}
$$

Hence, $\left\{S_{N+1}, P_{N+2}, P_{N+3}, \ldots\right\}$ is a Riesz system of projectors.

Remark 3. Projectors $P_{n}$ are one-dimensional for $n>N+1$ and $S_{N+1}$ has rank $N+1$. Therefore the system of root vectors of $T$ contains a Riesz basis $\left\{f_{n}\right\}_{n=1}^{\infty}$ with $f_{n}=\phi_{n}$ for $n>N+1$.

Proof. The proof is based on Criterion 1. The spectral projections $P_{n}^{0}$ of $A$ form a complete family of orthogonal projections, since $A$ is self-adjoint with discrete spectrum.

In order to apply Criterion [1, we have to find $N_{*}>N+2, N_{*} \in \mathbb{N}$, such that

$$
\forall f \in \mathcal{H}, \quad \sum_{n \geq N_{*}}^{\infty}\left\|P_{n}^{0}\left(P_{n}-P_{n}^{0}\right) f\right\|^{2} \leq \frac{1}{2}\|f\|^{2},
$$

so we take (in the notation of Criterion 1) $P_{0}:=\sum_{j=1}^{N_{*}-1} P_{j}^{0}$ and $Q_{0}=S_{N_{*}-1}$. The latter has the same rank as $P_{0}, c f$. Proposition 2 .

If $n>N+2$ and $z \in \Gamma_{n}$, then we have

$$
\begin{aligned}
& \left\|P_{n}^{0}\left(P_{n}-P_{n}^{0}\right) f\right\|^{2} \leq \frac{1}{4 \pi^{2}}\left\|\int_{\Gamma_{n}}\left((z-T)^{-1}-(z-A)^{-1}\right) f \mathrm{~d} z\right\|^{2} \\
& \leq \frac{1}{4 \pi^{2}}\left(\int_{\Gamma_{n}}\left\|(z-A)^{-\frac{1}{2}}\right\|\left\|(I-B(z))^{-1}\right\|\left\|B(z)(z-A)^{-\frac{1}{2}} f\right\||\mathrm{d} z|\right)^{2} \\
& \leq \frac{2}{\pi^{2}}\left(\int_{\Gamma_{n}}\left\|B(z)(z-A)^{-\frac{1}{2}} f\right\||\mathrm{d} z|\right)^{2}
\end{aligned}
$$

where we use the factorization of resolvent (6) and the bound $\|B(z)\| \leq 1 / 2$ for $z \in \Gamma_{n}, n>N$, cf. the proof of Proposition 2. Decomposing $f=\sum_{j=1}^{\infty} f_{j} \psi_{j} \in \mathcal{H}$ 
we obtain

$$
\begin{aligned}
\left\|B(z)(z-A)^{-\frac{1}{2}} f\right\|^{2} & =\sum_{k=1}^{\infty}\left|\left\langle B(z)(z-A)^{-\frac{1}{2}} \sum_{j=1}^{\infty} f_{j} \psi_{j}, \psi_{k}\right\rangle\right|^{2} \\
& \leq \sum_{k=1}^{\infty} \frac{1}{\left|\mu_{k}-z\right|}\left|\sum_{j=1}^{\infty} \frac{f_{j}}{z-\mu_{j}} b\left(\psi_{j}, \psi_{k}\right)\right|^{2} \\
& \leq M_{b}^{2} \sum_{k=1}^{\infty} \frac{1}{k^{2 \alpha}\left|\mu_{k}-z\right|}\left(\sum_{j=1}^{\infty} \frac{\left|f_{j}\right|}{j^{\alpha}\left|\mu_{j}-z\right|}\right)^{2} .
\end{aligned}
$$

For $n>N+2$, we select $z_{n}^{*} \in \Gamma_{n}$ for which the maximum of the integrand in the last integral in (14) is attained; notice that $\left\{z_{n}^{*}\right\}$ depends on $f$. Then we can continue estimates in (14),

$$
\left\|P_{n}^{0}\left(P_{n}-P_{n}^{0}\right) f\right\|^{2} \leq 2 M_{b}^{2} \sum_{k=1}^{\infty} \frac{1}{k^{2 \alpha}\left|\mu_{k}-z_{n}^{*}\right|}\left(\sum_{j=1}^{\infty} \frac{\left|f_{j}\right|}{j^{\alpha}\left|\mu_{j}-z_{n}^{*}\right|}\right)^{2} .
$$

We apply Lemma 5 on the first sum in (15),

$$
\left\|P_{n}^{0}\left(P_{n}-P_{n}^{0}\right) f\right\|^{2} \leq 2 M_{b}^{2} C(2 \alpha) \sigma_{2 \alpha}(n)\left(\sum_{j=1}^{\infty} \frac{\left|f_{j}\right|}{j^{\alpha}\left|\mu_{j}-z_{n}^{*}\right|}\right)^{2} .
$$

The final step is estimating the sum of $\left\|P_{n}^{0}\left(P_{n}-P_{n}^{0}\right) f\right\|^{2}$, starting at some $N_{1}>N+2, N_{1} \in \mathbb{N}$. We fix $\omega, 0<\omega<\min \{\alpha, 1 / 2\}$, and assume that $N_{1}$ is so large that $\sigma_{\alpha}(n) \leq \sigma_{\alpha}\left(N_{1}\right)$ and $\sigma_{2 \alpha}(n) n^{2 \omega} \leq \sigma_{2 \alpha}\left(N_{1}\right) N_{1}^{2 \omega}$ for $n \geq N_{1}$. Then, leaving aside the constant,

$$
\begin{aligned}
& \sum_{n=N_{1}}^{\infty} \sigma_{2 \alpha}(n)\left(\sum_{j=1}^{\infty} \frac{\left|f_{j}\right|}{j^{\alpha}\left|\mu_{j}-z_{n}^{*}\right|}\right)^{2} \leq \sigma_{2 \alpha}\left(N_{1}\right) N_{1}^{2 \omega} \sum_{n=N_{1}}^{\infty}\left(\sum_{j=1}^{\infty} \frac{\left|f_{j}\right|}{n^{\omega} j^{\alpha}\left|\mu_{j}-z_{n}^{*}\right|}\right)^{2} \\
& =\sigma_{2 \alpha}\left(N_{1}\right) N_{1}^{2 \omega}\|\mathcal{M} \tilde{f}\|_{\ell^{2}(\mathbb{N})}^{2},
\end{aligned}
$$

where $\mathcal{M}$ is an operator acting in $\ell^{2}(\mathbb{N})$ with matrix elements

$$
\begin{array}{ll}
\mathcal{M}_{n j}=0, & n<N_{1}, \\
\mathcal{M}_{n j}=\frac{1}{n^{\omega} j^{\alpha}\left|\mu_{j}-z_{n}^{*}\right|}, & n \geq N_{1} .
\end{array}
$$

and $\tilde{f}=\left\{\left|f_{n}\right|\right\}_{n \in \mathbb{N}} \in \ell^{2}(\mathbb{N})$.

We intend to bound $\|\mathcal{M}\|$ using the Schur test, cf. [25] or [12, Sec. 3], [16, Thm. 5.2]. To this end we estimate the following sums by applying Lemma 5

$$
\begin{aligned}
& \sum_{j=1}^{\infty}\left|\mathcal{M}_{n j}\right|=\sum_{j=1}^{\infty} \frac{1}{n^{\omega} j^{\alpha}\left|\mu_{j}-z_{n}^{*}\right|} \leq \frac{1}{n^{\omega}} C(\alpha) \sigma_{\alpha}(n) \leq C(\alpha) \frac{\sigma_{\alpha}\left(N_{1}\right)}{N_{1}^{\omega}}, \\
& \sum_{n=1}^{\infty}\left|\mathcal{M}_{n j}\right| \leq \frac{1}{j^{\alpha}} C(\omega) \sigma_{\omega}(j) \leq \frac{C(\omega)}{\omega e}
\end{aligned}
$$

where we use (17) and that $N_{1}$ is such that $\sigma_{\alpha}(n) \leq \sigma_{\alpha}\left(N_{1}\right)$. The Schur test then yields

$$
\|\mathcal{M}\|^{2} \leq \frac{C(\alpha) C(\omega)}{\omega e} \frac{\sigma_{\alpha}\left(N_{1}\right)}{N_{1}^{\omega}}
$$


Therefore, since $\omega<\alpha$,

$$
\sum_{n \geq N_{1}}^{\infty}\left\|P_{n}^{0}\left(P_{n}-P_{n}^{0}\right) f\right\|^{2} \leq \frac{2 M_{b}^{2} C(2 \alpha) C(\alpha) C(\omega)}{\omega e} \sigma_{2 \alpha}\left(N_{1}\right) \sigma_{\alpha}\left(N_{1}\right) N_{1}^{\omega}\|f\|^{2},
$$

which proves the existence of sought $N_{*}$ and Theorem 3 .

\section{TeChnical LEMmas}

We collect technical results, mainly estimates on the sums appearing in the proofs of main results. At first we explain in details the form subordination of the perturbation.

Lemma 4. Let $A$ and $b$ satisfy (1) and (4). Then there exist $0 \leq p<1$ and $C>0$ such that

$$
\forall f \in \operatorname{Dom}(a),|b(f, f)| \leq C(a(f, f))^{p}\|f\|^{2(1-p)},
$$

i.e. $b$ is p-subordinated to $a$. Moreover, for $\alpha \leq 1 / 2$, $p$ can be selected as $1-2 \alpha+\tau$, with $\tau>0$ arbitrarily small. If $\alpha>1 / 2$, then $b$ is bounded.

Proof. Writing $f=\sum_{j=1}^{\infty} f_{j} \psi_{j}$, we get $(0 \leq \beta<1 / 2)$

$$
\begin{aligned}
|b(f, f)| & =\left|\sum_{j, k=1}^{\infty} \bar{f}_{j} f_{k} b\left(\psi_{j}, \psi_{k}\right)\right| \leq M_{b}\left(\sum_{j=1}^{\infty} \frac{\left|f_{j}\right|}{j^{\alpha}}\right)^{2}=M_{b}\left(\sum_{j=1}^{\infty}\left|f_{j}\right| j^{\beta} \frac{1}{j^{\alpha+\beta}}\right)^{2} \\
& \leq M_{b}\left\|A^{\beta} f\right\|^{2} \sum_{j=1}^{\infty} \frac{1}{j^{2(\alpha+\beta)}} \leq M_{b}(a(f, f))^{2 \beta}\|f\|^{2(1-2 \beta)} \sum_{j=1}^{\infty} \frac{1}{j^{2(\alpha+\beta)}},
\end{aligned}
$$

where we used the Hölder inequality in the last step. For $\alpha \leq 1 / 2$, we select $\beta=1 / 2-\alpha+\tau / 2, \tau>0$ and we receive the claim with $p=1-2 \alpha+\tau$. For $\alpha>1 / 2$, we can take $\beta=0$ and therefore $b$ is bounded.

Lemma 5. Let $n \in \mathbb{N}, n>1$, Re $z_{n} \in\left[\mu_{n}-1 / 2, \mu_{n+1}-1 / 2\right]$, and $z_{n} \notin \Pi_{n}$, where $\Pi_{n}$ is defined in (ㅈ) and $\gamma>0$. Then

$$
\sum_{k=1}^{\infty} \frac{1}{k^{\gamma}\left|\mu_{k}-z_{n}\right|} \leq C(\gamma) \sigma_{\gamma}(n)
$$

where $C(\gamma)$ does not depend on $n$ and

$$
\sigma_{\gamma}(n):= \begin{cases}n^{-\gamma} \log n, & \gamma \leq 1 \\ n^{-1}, & \gamma>1\end{cases}
$$

Proof. Using $\left|\mu_{k}-z_{n}\right| \geq|k-n| / 2$ for $k \notin\{n, n+1\},\left|\mu_{n}-z_{n}\right| \geq 1 / 2$, and $\left|\mu_{n+1}-z_{n}\right| \geq 1 / 2$, we obtain

$$
\sum_{k=1}^{\infty} \frac{1}{k^{\gamma}\left|\mu_{k}-z_{n}\right|} \leq 2\left(\sum_{k=1}^{n-1} \frac{1}{k^{\gamma}(n-k)}+\frac{2}{n^{\gamma}}+\sum_{k=n+2}^{\infty} \frac{1}{k^{\gamma}(k-n)}\right) .
$$

If $f$ is a convex non-negative function in interval $[1, p]$, then

$$
\int_{1}^{p} f(x) \mathrm{d} x \leq \sum_{i=1}^{p} f(i) \leq f(1)+f(p)+\int_{1}^{p} f(x) \mathrm{d} x .
$$

Therefore the first term on the right in (16) can be estimated as

$$
\sum_{k=1}^{n-1} \frac{1}{k^{\gamma}(n-k)} \leq \frac{1}{n-1}+\frac{1}{(n-1)^{\gamma}}+\int_{1}^{n-1} \frac{\mathrm{d} x}{x^{\gamma}(n-x)}
$$


Splitting the integral we obtain

$$
\begin{aligned}
\int_{\frac{n}{2}}^{n-1} \frac{\mathrm{d} x}{x^{\gamma}(n-x)} & \leq \frac{2^{\gamma}}{n^{\gamma}} \int_{\frac{n}{2}}^{n-1} \frac{\mathrm{d} x}{n-x} \leq 2^{\gamma} \frac{\log n}{n^{\gamma}}, \\
\int_{1}^{\frac{n}{2}} \frac{\mathrm{d} x}{x^{\gamma}(n-x)} & \leq \frac{2}{n} \int_{1}^{\frac{n}{2}} \frac{\mathrm{d} x}{x^{\gamma}}
\end{aligned}
$$

where depending on $\gamma$

$$
\frac{2}{n} \int_{1}^{\frac{n}{2}} \frac{\mathrm{d} x}{x^{\gamma}} \leq \begin{cases}2^{\gamma}(1-\gamma)^{-1} n^{-\gamma}, & \gamma<1 \\ 2 n^{-1} \log n, & \gamma=1 \\ 2(\gamma-1)^{-1} n^{-1}, & \gamma>1\end{cases}
$$

The third term in (16) is split as well

$$
\sum_{k=n+2}^{\infty} \frac{1}{k^{\gamma}(k-n)}=\sum_{k=n+2}^{2 n} \frac{1}{k^{\gamma}(k-n)}+\sum_{k=2 n+1}^{\infty} \frac{1}{k^{\gamma}(k-n)}
$$

and estimated as

$$
\begin{aligned}
& \sum_{k=n+2}^{2 n} \frac{1}{k^{\gamma}(k-n)} \leq \frac{1}{n^{\gamma}} \sum_{k=n+2}^{2 n} \frac{1}{k-n} \leq \frac{1}{n^{\gamma}} \int_{n+1}^{2 n} \frac{\mathrm{d} x}{x-n} \leq \frac{\log n}{n^{\gamma}} \\
& \sum_{k=2 n+1}^{\infty} \frac{1}{k^{\gamma}(k-n)} \leq 2 \sum_{k=2 n+1}^{\infty} \frac{1}{k^{\gamma+1}} \leq 2 \int_{2 n}^{\infty} \frac{\mathrm{d} x}{x^{\gamma+1}} \leq \frac{2^{1-\gamma}}{\gamma} \frac{1}{n^{\gamma}}
\end{aligned}
$$

where we used $k-n>k / 2$ in the second estimate.

Combing all the inequalities and using for $\gamma=1+\beta>1$ that

$$
\frac{\log n}{n^{\beta}} \leq \max _{x \geq 0} \frac{x}{e^{\beta x}}=\frac{1}{\beta e},
$$

we obtain the claim.

Lemma 6. Let $h>1$. Then

$$
\sum_{k=1}^{\infty} \frac{1}{k^{\gamma}(k+h)} \leq D(\gamma) \tau_{\gamma}(h)
$$

where $D(\gamma)$ does not depend on $h$ and

$$
\tau_{\gamma}(h):= \begin{cases}h^{-\gamma}, & \text { if } \gamma<1 \\ h^{-1} \log h, & \text { if } \gamma=1 \\ h^{-1}, & \text { if } \gamma>1\end{cases}
$$

Proof. Proof is analogous to the one of Lemma 5]

Remark 4. A careful analysis of our proof and proper adjustments in the proofs of technical lemmas [5 and [6 and in the inequalities related to (15) show that we can weaken Condition (4) in Theorem 3 by assuming only that

(4A) $\exists \beta>\frac{3}{2}, \quad \exists M_{b}>0, \quad \forall m, n \in \mathbb{N}, \quad\left|b\left(\psi_{m}, \psi_{n}\right)\right| \leq \frac{M_{b}}{(\log (m+1) \log (n+1))^{\beta}}$. 


\section{ExAmples}

We start with the analysis of perturbations of the harmonic oscillator in $L^{2}(\mathbb{R})$ :

$$
\begin{aligned}
A & =-\frac{\mathrm{d}^{2}}{\mathrm{~d} x^{2}}+x^{2}, & \operatorname{Dom}(A) & =\left\{\psi \in W^{2,2}(\mathbb{R}): x^{2} \psi \in L^{2}(\mathbb{R})\right\}, \\
a(\psi, \psi) & =\left\|\psi^{\prime}\right\|^{2}+\|x \psi\|^{2}, & \operatorname{Dom}(a) & =\left\{\psi \in W^{1,2}(\mathbb{R}): x \psi \in L^{2}(\mathbb{R})\right\} .
\end{aligned}
$$

Eigenvalues of $A$ are $\mu_{n}=2 n+1, n \in \mathbb{N}_{0}$, and eigenfunctions are Hermite functions

$$
h_{n}(x)=\frac{1}{\left(2^{n} n ! \sqrt{\pi}\right)^{\frac{1}{2}}} e^{-\frac{x^{2}}{2}} H_{n}(x), \quad n=0,1,2, \ldots
$$

5.1. $\delta$ potential. The first example is the perturbation by the $\delta$ potential placed in $x_{0}$ with coupling $\nu \in \mathbb{C}$, more precisely

$$
b_{1}(\phi, \psi)=\nu \phi\left(x_{0}\right) \overline{\psi\left(x_{0}\right)}, \quad \nu \in \mathbb{C}, \quad \operatorname{Dom}\left(b_{1}\right)=W^{1,2}(\mathbb{R}) .
$$

In the following, we estimate $\left|b_{1}\left(h_{m}, h_{n}\right)\right|=|\nu|\left|h_{m}\left(x_{0}\right)\right|\left|h_{n}\left(x_{0}\right)\right|$.

If $x_{0}=0$, the values $h_{n}(0)$ read, $c f$. [1, Eq.22.4.8, Eq.22.2.14],

$$
h_{2 n-1}(0)=0, \quad h_{2 n}(0)=\frac{(-1)^{n}((2 n) !)^{\frac{1}{2}}}{\pi^{\frac{1}{4}} 2^{n} n !} .
$$

Using the Stirling formula for the factorial,

$$
n !=\sqrt{2 \pi n}\left(\frac{n}{e}\right)^{n} e^{\lambda_{n}}, \quad \frac{1}{12 n+1}<\lambda_{n}<\frac{1}{12 n},
$$

cf. [1, Eq.6.1.38], [13, §2.9], yields

$$
\left|h_{2 n}(0)\right|=\frac{1+\mathcal{O}\left(\frac{1}{n}\right)}{\pi^{\frac{1}{2}} n^{\frac{1}{4}}},
$$

hence the perturbation $b_{1}$ satisfies condition (4) with $\alpha=1 / 4$ if $x_{0}=0$.

Further analysis, $c f$. [6, p.700] and further references in [3], shows that

$$
\left|h_{n}(x)\right| \leq C \begin{cases}\left(N^{\frac{1}{2}}\left(N^{\frac{1}{2}}-x\right)\right)^{-\frac{1}{4}}, & 0 \leq x \leq N^{\frac{1}{2}}-N^{-\frac{1}{6}}, \\ N^{-\frac{1}{12}}, & N^{\frac{1}{2}}-N^{-\frac{1}{6}} \leq x \leq N^{\frac{1}{2}}+N^{-\frac{1}{6}}, \\ \frac{\exp \left(-\xi N^{\frac{1}{4}}\left(x-N^{\frac{1}{2}}\right)^{\frac{3}{2}}\right)}{\left(N^{\frac{1}{2}}\left(x-N^{\frac{1}{2}}\right)\right)^{\frac{1}{4}}}, & N^{\frac{1}{2}}+N^{-\frac{1}{6}} \leq x \leq(2 N)^{\frac{1}{2}}, \\ \exp \left(-\xi x^{2}\right), & x \geq(2 N)^{\frac{1}{2}},\end{cases}
$$

where $N=2 n+1$ and $\xi>0$. Therefore we can apply our results, since $\left|h_{n}\left(x_{0}\right)\right| \leq$ $C n^{-1 / 4}$ when $n$ is sufficiently large, e.g. if $N^{1 / 2} \geq x_{0}+1$.

5.2. Function potential $V$. We consider a form generated by a function potential

$$
b_{2}(\phi, \psi):=\langle V \phi, \psi\rangle \text {. }
$$

At first let $V \in L^{p}(\mathbb{R}), 1 \leq p<\infty$. The condition (44) holds for $b_{2}$, since

$$
\begin{aligned}
\left|\left\langle V h_{m}, h_{n}\right\rangle\right| & \leq\|V\|_{L^{p}}\left\|h_{m} h_{n}\right\|_{L^{q}} \leq\|V\|_{L^{p}}\left\|h_{m} h_{n}\right\|_{L^{\infty}}^{\frac{1}{p}}\left\|h_{m} h_{n}\right\|_{L^{1}}^{\frac{1}{q}} \\
& \leq C(m n)^{-\frac{1}{12 p}}, \quad m, n \in \mathbb{N},
\end{aligned}
$$

where $1 / p+1 / q=1$ and we use $\left\|h_{m} h_{n}\right\|_{L^{1}} \leq 1$ and (3). Hence the statement of Theorem 3 holds.

A next step is to take $V \in L(p, \tau)$, where

$$
L(p, \tau):=\left\{v:\left(1+|x|^{2}\right)^{-\frac{\tau}{2}}|v(x)| \in L^{p}(\mathbb{R})\right\}, \quad 1 \leq p<\infty, \tau \geq 0,
$$


is introduced in $[3]$; notice that $L^{p}(\mathbb{R})$ is a special case for $\tau=0$. It has been showed in [3] that for $2 \leq p<\infty$ and $n \in \mathbb{N}, n>1$,

$$
\left\|V h_{n}\right\| \leq C \begin{cases}n^{\frac{\tau}{2}+t(p)}, & p \neq 4 \\ n^{\frac{\tau}{2}-\frac{1}{8}} \log n, & p=4\end{cases}
$$

with

$$
t(p)= \begin{cases}-\frac{1}{6}\left(1-\frac{1}{p}\right), & 2 \leq p<4 \\ -\frac{1}{2 p}, & 4 \leq p<\infty\end{cases}
$$

see [3, Lem.5.2] for details. Hence the condition (2) holds for $V \in L(p, \tau)$ if $2 \leq$ $p<\infty$ and $\tau / 2+t(p)<0$. (The condition (2) holds for $V$ also if $\tau / 2+t(p) \leq 0$, $(p, \tau) \neq(4,1 / 4)$, see [3, Prop.5.4].) Therefore the eigensystem of $T=A+V$ contains a Riesz basis, $c f$. [3, Thm.1.3]. Now we can explain this result of [3] as a corollary of Theorem 3 , indeed

$$
\left|b_{2}\left(h_{m}, h_{n}\right)\right|=\left|\left\langle V h_{m}, h_{n}\right\rangle\right| \leq \min \left(\left\|V h_{m}\right\|,\left\|V h_{n}\right\|\right) \leq\left\|V h_{m}\right\|^{\frac{1}{2}}\left\|V h_{n}\right\|^{\frac{1}{2}}
$$

and the inequalities (24) imply (4) with some $\alpha>0$.

The approach with forms enables us to include potentials $V \in L(p, \tau)$ with $1 \leq p<2$ and a suitable $\tau$ in our analysis and obtain the following claim.

Theorem 7. Let $A$ be the harmonic oscillator, cf. (18), and $V \in L(p, \tau)$ with $1 \leq p<\infty, \tau \geq 0$ and $\tau / 4+t(2 p)<0$, where $t(p)$ is defined in (25). Then the statement of Theorem 3 holds for $T=A+V$ defined as a sum of forms.

Proof. The proof is based on the inequality

$$
\left\|h_{n}(x)\left(1+|x|^{2}\right)^{\frac{\tilde{\tau}}{2}}\right\|_{L^{\tilde{q}}} \leq C \begin{cases}n^{\frac{\tilde{\tau}}{2}+t(\tilde{p})}, & \tilde{p} \neq 4, \\ n^{\frac{\tilde{\tau}}{2}-\frac{1}{8}} \log n, & \tilde{p}=4,\end{cases}
$$

where $1 / \tilde{q}+1 / \tilde{p}=1 / 2$ and $n \in \mathbb{N}, n>1$, proved in [2, Sec.6.2], [3, Eq.(41)-(46)] using (21). If $V \in L(p, \tau)$, then

$$
\begin{aligned}
\left|b_{2}\left(h_{m}, h_{n}\right)\right| & \leq \int_{\mathbb{R}}|V(x)|\left(1+|x|^{2}\right)^{-\frac{\tau}{2}}\left|h_{m}(x)\right|\left|h_{n}(x)\right|\left(1+|x|^{2}\right)^{\frac{\tau}{2}} \mathrm{~d} x \\
& \leq\left\|V(x)\left(1+|x|^{2}\right)^{-\frac{\tau}{2}}\right\|_{L^{p}}\left\|h_{m}(x) h_{n}(x)\left(1+|x|^{2}\right)^{\frac{\tau}{2}}\right\|_{L^{q}} \\
& \leq C\left\|h_{m}(x)\left(1+|x|^{2}\right)^{\frac{\tau}{4}}\right\|_{L^{2 q}}\left\|h_{n}(x)\left(1+|x|^{2}\right)^{\frac{\tau}{4}}\right\|_{L^{2 q}},
\end{aligned}
$$

where $1 / p+1 / q=1$. The inequality (26) with $\tilde{\tau}=\tau / 2$ and $\tilde{q}=2 q$ yields, for all $n \in \mathbb{N}, n>1$,

$$
\left\|h_{n}(x)\left(1+|x|^{2}\right)^{\frac{\tau}{4}}\right\|_{L^{2 q}} \leq C \begin{cases}n^{\frac{\tau}{4}+t(2 p)}, & p \neq 2 \\ n^{\frac{\tau}{4}-\frac{1}{8}} \log n, & p=2 .\end{cases}
$$

Therefore the condition (4) holds for $b_{2}$ if $\tau / 4+t(2 p)<0$ and Theorem 3 can be applied.

5.3. Singular potential with compact support. Another example is a form generated by a singular potential $V \in W^{-s, 2}(\mathbb{R}), 0<s<1 / 2$, with a compact support, contained in an interval $[-p, p]$,

$$
b_{3}(\phi, \psi):=(V, \phi \bar{\psi}) \text {. }
$$

For $n$ sufficiently large, e.g. $N^{1 / 2} \geq 2 p+1$, with $N=2 n+1$, we have

$$
\left|h_{n}(x)\right| \leq C n^{-\frac{1}{4}}, \quad\left|h_{n}^{\prime}(x)\right| \leq C n^{\frac{1}{4}}, \quad x \in[-3 p / 2,3 p / 2] .
$$


These estimates follow from (21) and the relation for the derivative of Hermite functions

$$
h_{n}^{\prime}(x)=\sqrt{\frac{n}{2}} h_{n-1}(x)+\sqrt{\frac{n+1}{2}} h_{n+1}(x) .
$$

Therefore, for $m, n \in \mathbb{N}$,

$$
\left|b_{3}\left(h_{m}, h_{n}\right)\right|=\left|\left(V, h_{m} h_{n}\right)\right| \leq C\left\|h_{m} h_{n}\right\|_{L^{2}((-p, p))}^{1-s}\left\|h_{m} h_{n}\right\|_{W^{1,2}((-p, p))}^{s} \leq C(m n)^{\frac{2 s-1}{4}}
$$

and the condition (4) is satisfied for $s<1 / 2$. Hence the statement of Theorem 3 holds.

The assumption on the compact support of singular potential can be omitted, but the range of $s$ is more restrictive. Namely, if $V \in W^{-s, 2}(\mathbb{R}), 0<s<1 / 11$, then the statement of Theorem 3 holds. The proof of this claim is analogous, but we use (3) and (28) or more general inequalities for weighted polynomials, cf. 21, 34.

Remark 5. The condition (4) puts restrictions on the system of linear functionals of $V$. Therefore, if we can represent $V$ as a finite sum of potentials $\left\{V_{j}\right\}$ in such a way that each $V_{j}$ satisfies condition (4), then $V$ satisfies the condition (4) as well and the statement of Theorem 3 on the Riesz basisness of eigensystem of $T=A+V$ holds. In particular, the following is true.

Corollary 8. Let $A$ be the harmonic oscillator, cf. (18). If $V=V_{1}+V_{2}+V_{3}$, where $V_{3} \in W^{-s, 2}(\mathbb{R}), 0<s<1 / 2$, with compact support, $V_{2} \in L(p, \tau)$ with $1 \leq p<\infty$, $\tau \geq 0$ and $\tau / 4+t(2 p)<0$, cf. (23), (25), and $V_{1} \in L^{1}(\mathbb{R})$, then the statement of Theorem 3 holds for $T=A+V$ defined as a sum of forms.

5.4. Infinite number of $\delta$-potentials. We can go to infinite sums $V=\sum_{j} V_{j}$, but then we have to watch carefully the behavior of constants $M_{j}$ in (4), i.e.

$$
\left|\left\langle V_{j} h_{m}, h_{n}\right\rangle\right| \leq \frac{M_{j}}{m^{\alpha} n^{\alpha}} .
$$

To illustrate this point we consider the infinite number of $\delta$ potentials.

Example 9. Let $A$ be the harmonic oscillator, cf. (18), and let

$$
b_{4}(\phi, \psi)=\sum_{k=-\infty}^{\infty} \nu_{k} \phi\left(p_{k}\right) \overline{\psi\left(p_{k}\right)}
$$

where points $\left\{p_{k}\right\}_{k \in \mathbb{Z}}$ and coupling constants $\left\{\nu_{k}\right\}_{k \in \mathbb{Z}}$ satisfy $p_{k}=(\operatorname{sgn} k)|k|^{\gamma}, 0<$ $\gamma \leq 1$, and $\nu_{k}=\mathcal{O}\left(|k|^{-\beta}\right), \beta \geq 0$, respectively. If $\beta+\gamma>1$, then $b_{4}$ satisfies the condition (4) and therefore the statement of Theorem 3 holds.

Proof. We intend to determine the relation between $\beta$ and $\gamma$ guaranteeing that $b_{4}$ satisfies the assumption (4). In the first step, we use Hölder inequality and the fact that Hermite functions are either even or odd and obtain

$$
\left|b_{4}\left(h_{m}, h_{n}\right)\right| \leq 2\left(\sum_{k=0}^{\infty}\left|\nu_{k}\right|\left|h_{m}\left(p_{k}\right)\right|^{2}\right)^{\frac{1}{2}}\left(\sum_{k=0}^{\infty}\left|\nu_{k}\right|\left|h_{n}\left(p_{k}\right)\right|^{2}\right)^{\frac{1}{2}} .
$$

The estimates are based on the behavior of Hermite functions (21) and are divided into four parts. For $k \leq K_{1}$ with $K_{1} \in \mathbb{N}$ such that $\left(N^{1 / 2}-N^{-1 / 6}\right)^{1 / \gamma}-1 \leq K_{1} \leq$ $\left(N^{1 / 2}-N^{-1 / 6}\right)^{1 / \gamma}$, we have

$$
\sum_{k=1}^{K_{1}} \frac{N^{-\frac{1}{4}}}{k^{\beta}\left(N^{\frac{1}{2}}-k^{\gamma}\right)^{\frac{1}{2}}} \leq \int_{1}^{K_{1}} \frac{N^{-\frac{1}{4}} \mathrm{~d} x}{x^{\beta}\left(N^{\frac{1}{2}}-x^{\gamma}\right)^{\frac{1}{2}}}+\frac{2 N^{-\frac{1}{4}}}{p_{*}^{\beta}\left(N^{\frac{1}{2}}-p_{*}^{\gamma}\right)^{\frac{1}{2}}},
$$


where $1 \leq p_{*} \leq K_{1}$ is such that the maximum of $x^{-\beta}\left(N^{1 / 2}-x^{\gamma}\right)^{-1 / 2}$ is attained at $x=p_{*}$. By a very crude estimate without searching for the actual value of $p_{*}$, the second term is $O\left(N^{-1 / 6}\right)$. The substitution $x^{\gamma}=N^{1 / 2} y$ in the integral leads to

$$
\int_{1}^{K_{1}} \frac{N^{-\frac{1}{4}} \mathrm{~d} x}{x^{\beta}\left(N^{\frac{1}{2}}-x^{\gamma}\right)^{\frac{1}{2}}} \leq \frac{N^{\frac{1-\beta-\gamma}{2 \gamma}}}{\gamma} \int_{N^{-\frac{1}{2}}}^{1} \frac{y^{\frac{1-\beta}{\gamma}-1} \mathrm{~d} y}{(1-y)^{\frac{1}{2}}} .
$$

As $N \rightarrow+\infty$ the integral is $\mathcal{O}(1)$ for $0<\beta<1, \mathcal{O}(\log N)$ for $\beta=1$, and $\mathcal{O}\left(N^{\frac{\beta-1}{2 \gamma}}\right)$ for $\beta>1$. Taking into account the behavior of the prefactor, assumption (4) is satisfied if $\beta>1-\gamma$.

In the second part, we have

$$
\sum_{k=K_{1}+1}^{K_{2}} \frac{N^{-\frac{1}{6}}}{k^{\beta}} \leq \frac{N^{-\frac{1}{6}}}{\left(K_{1}+1\right)^{\beta}}+\int_{K_{1}+1}^{K_{2}} \frac{N^{-\frac{1}{6}} \mathrm{~d} x}{x^{\beta}},
$$

where $\left(N^{1 / 2}+N^{-1 / 6}\right)^{1 / \gamma}-1 \leq K_{2} \leq\left(N^{1 / 2}+N^{-1 / 6}\right)^{1 / \gamma}$. The first term is $\mathcal{O}\left(N^{\frac{-\gamma-3 \beta}{6 \gamma}}\right)$, giving no condition on $\beta$. For $\beta \neq 1$, the integral can be estimated as

$$
\begin{aligned}
\int_{K_{1}+1}^{K_{2}} \frac{N^{-\frac{1}{6}} \mathrm{~d} x}{x^{\beta}} & \leq \int_{\left(N^{\frac{1}{2}}-N^{-\frac{1}{6}}\right)^{\frac{1}{\gamma}}}^{\left(N^{\frac{1}{2}}+N^{-\frac{1}{6}}\right)^{\frac{1}{\gamma}}} \frac{N^{-\frac{1}{6}} \mathrm{~d} x}{x^{\beta}} \\
& \leq \frac{N^{-\frac{1}{6}}}{|1-\beta|}\left|\left(N^{\frac{1}{2}}+N^{-\frac{1}{6}}\right)^{\frac{1-\beta}{\gamma}}-\left(N^{\frac{1}{2}}-N^{-\frac{1}{6}}\right)^{\frac{1-\beta}{\gamma}}\right| \\
& =\frac{N^{\frac{3-3 \beta-\gamma}{6 \gamma}}}{|1-\beta|}\left|\frac{2(1-\beta)}{\gamma} N^{-\frac{2}{3}}+\mathcal{O}\left(N^{-\frac{4}{3}}\right)\right|=\mathcal{O}\left(N^{\frac{3-3 \beta-5 \gamma}{6 \gamma}}\right)
\end{aligned}
$$

For $\beta=1$ the integral is $o\left(N^{-1 / 6}\right)$. Therefore the condition on $\beta \operatorname{reads} \beta>1-5 \gamma / 3$, which is less restrictive than $\beta>1-\gamma$ from the first part. Using analogous estimates, it can be verified that the conditions on $\beta$ from the third and fourth part are also weaker. Therefore, we will make the assumption $\beta>1-\gamma$ to guarantee that the condition (4) holds and therefore the statement of Theorem 3 holds.

5.5. Perturbations of anharmonic oscillators. Now we make a few remarks to extend the results of 4 . We perturb self-adjoint operators in $L^{2}(\mathbb{R})$ :

$$
\begin{aligned}
A & =-\frac{\mathrm{d}^{2}}{\mathrm{~d} x^{2}}+|x|^{\beta}, \quad \beta \geq 2, \\
\operatorname{Dom}(A) & =\left\{\psi \in W^{2,2}(\mathbb{R}):|x|^{\beta} \psi \in L^{2}(\mathbb{R})\right\}, \\
a(\psi, \psi) & =\left\|\psi^{\prime}\right\|^{2}+\left\||x|^{\frac{\beta}{2}} \psi\right\|^{2}, \\
\operatorname{Dom}(a) & =\left\{\psi \in W^{1,2}(\mathbb{R}):|x|^{\frac{\beta}{2}} \psi \in L^{2}(\mathbb{R})\right\} .
\end{aligned}
$$

The spectrum of $A$ is discrete and eigenvalues are simple. Moreover, eigenvalues $\mu_{n}$ and normalized eigenfunctions $\phi_{n}$ satisfy

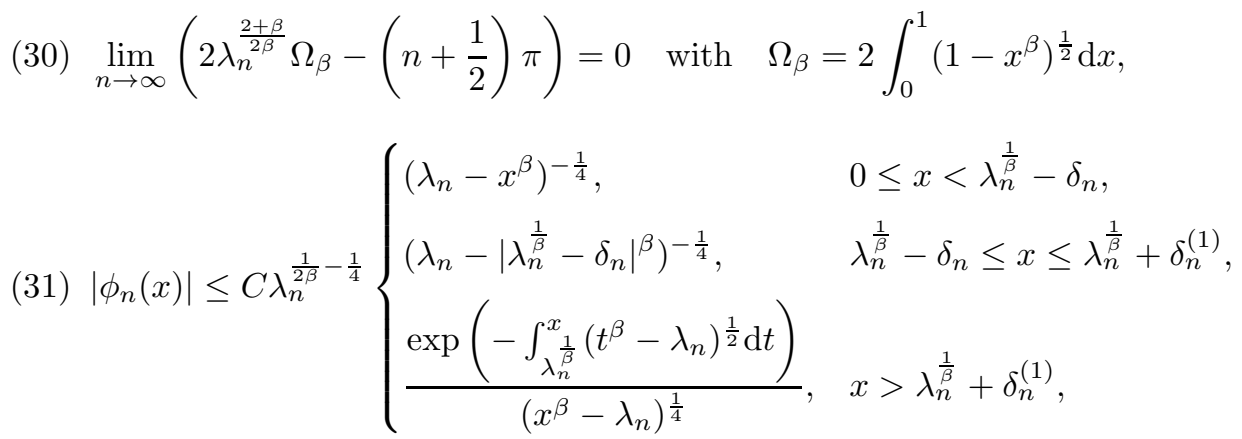


where $\delta_{n}$ and $\delta_{n}^{(1)}$ are defined by

$$
\int_{\lambda_{n}^{\frac{1}{\beta}}-\delta_{n}}^{\lambda_{n}^{\frac{1}{\beta}}}\left(\lambda_{n}-x^{\beta}\right)^{\frac{1}{2}} \mathrm{~d} x=1, \quad \int_{\lambda_{n}^{\frac{1}{\beta}}}^{\lambda_{n}^{\frac{1}{\beta}}+\delta_{n}^{(1)}}\left(x^{\beta}-\lambda_{n}\right)^{\frac{1}{2}} \mathrm{~d} x=1
$$

and satisfy $\delta_{n}, \delta_{n}^{(1)}=\mathcal{O}\left(\lambda_{n}^{-\frac{1-\beta}{3 \beta}}\right)$ and $\delta_{n}^{-1},\left(\delta_{n}^{(1)}\right)^{-1}=\mathcal{O}\left(\lambda_{n}^{\frac{1-\beta}{3 \beta}}\right)$, see 30, 14, 31, Sec.22.27] and [24] for more details.

The growth condition on eigenvalues implies the existence of constant $C>0$ and $N \in \mathbb{N}$, both depending on $\beta$, such that for all $n>N$

$$
\lambda_{n+1}-\lambda_{n} \geq C n^{\alpha-1}, \quad \text { with } \quad \alpha=\frac{2 \beta}{\beta+2},
$$

cf. [4, Sec.8.2]. Therefore, if $\beta \geq 2$, the growth condition on $\mu_{n}$ in (11) is satisfied.

As a perturbation we consider form $b_{2}, c f$. (22), generated by a function potential $V \in L(p, \tau), c f$. (23), and formulate an analogous result to Theorem 7 for harmonic oscillator.

Theorem 10. Let $A$ be the anharmonic oscillator, cf. (29), and $V \in L(p, \tau)$ with $1 \leq p<\infty, \tau \geq 0$ and $\tau /(\beta+2)+t(2 p, \beta)<0$, where

$$
t(p, \beta):= \begin{cases}-\frac{1}{6}\left(1-\frac{4(\beta-1)}{\beta+2} \frac{1}{p}\right), & 1 \leq p<2, \\ -\frac{2}{\beta+2} \frac{1}{p}, & 2 \leq p \leq \infty,\end{cases}
$$

Then the statement of Theorem 3 holds for $T=A+V$ defined as a sum of forms.

Proof. The proof follows the lines of the one of Theorem [7, but the estimate for $\phi_{n}, n \in \mathbb{N}, n>1$, is used,

$$
\left\|\phi_{n}(x)\left(1+|x|^{2}\right)^{\frac{\tilde{\tau}}{2}}\right\|_{L^{\tilde{q}}} \leq C \begin{cases}n^{\frac{2}{\beta+2}\left(\tilde{\tau}-\frac{1}{\tilde{p}}\right)}, & 2 \leq \tilde{p}<4, \\ n^{\frac{2}{\beta+2}\left(\tilde{\tau}-\frac{1}{4}\right)} \log n, & \tilde{p}=4, \\ n^{-\frac{1}{6}+\frac{2}{\beta+2}\left(\tilde{\tau}+\frac{\beta-1}{3 \tilde{p}}\right)}, & \tilde{p}>4,\end{cases}
$$

where $1 / \tilde{q}+1 / \tilde{p}=1 / 2$. If $V \in L(p, \tau)$, then the same procedure as in (27) gives

$$
\left|b_{2}\left(\phi_{m}, \phi_{n}\right)\right| \leq C\left\|\phi_{m}(x)\left(1+|x|^{2}\right)^{\frac{\tau}{4}}\right\|_{L^{2 q}}\left\|\phi_{n}(x)\left(1+|x|^{2}\right)^{\frac{\tau}{4}}\right\|_{L^{2 q}},
$$

where $1 / p+1 / q=1$, and the claim is then obtained from inequalities (34) with $\tilde{\tau}=\tau / 2$ and $\tilde{q}=2 q$, i.e. $\tilde{p}=2 p$.

\section{Conclusions}

The positive results, i.e. the claims that the eigensystem of $T$ contains a Riesz basis, are obtained if the local subordination condition in the sense of operators (2), used in [3, 4, 26, or in the sense of forms (4) is satisfied. The usual subordination condition in the sense of operators or forms, $c f$. (5), is used in previous works 20, [5, 32 where no claims on the Riesz basisness for the perturbations of the harmonic oscillator are given (except a weaker result of Riesz basis of subspaces for bounded perturbations, $c f$. [5]). As it follows from [24, Thm.2.6], eq. (2.18), the example

$$
-\frac{\mathrm{d}^{2}}{\mathrm{~d} x^{2}}+x^{2}+2 \mathrm{i} x
$$

shows that the subordination does not help to claim even the basisness of eigensystem, since in this case the perturbation $2 \mathrm{i} x$ is subordinated, however, the norms of Riesz projections $P_{n}$ grows in $n$, namely

$$
\lim _{n \rightarrow \infty} \frac{1}{\sqrt{n}} \log \left\|P_{n}\right\|=2^{\frac{3}{2}}
$$


In fact, various rates of spectral projection growth can be obtained by subordinated perturbations of harmonic and anharmonic oscillators, $c f$. 24].

\section{REFERENCES}

[1] Abramowitz, M., And Stegun, I. A., Eds. Handbook of mathematical functions with formulas, graphs, and mathematical tables. U.S. Government Printing Office, Washington, D.C., 1964.

[2] AdDucI, J. Perturbations of selfadjoint operators with discrete spectrum. PhD thesis, 2011. Thesis (Ph.D.)-The Ohio State University.

[3] Adduci, J., And Mityagin, B. Eigensystem of an $L^{2}$-perturbed harmonic oscillator is an unconditional basis. Central European Journal of Mathematics 10 (2012), 569-589.

[4] Adduci, J., And Mityagin, B. Root System of a Perturbation of a Selfadjoint Operator with Discrete Spectrum. Integral Equations and Operator Theory 73 (2012), 153-175.

[5] Agranovich, M. S. On series in root vectors of operators defined by forms with a selfadjoint principal part. Funktsional'nyॅ Analiz i ego Prilozheniya 28 (1994), 1-21, 95. Translation in Functional Analysis and Its Applications 28 (1994), 151-167.

[6] Askey, R., And Wainger, S. Mean Convergence of Expansions in Laguerre and Hermite Series. American Journal of Mathematics 87 (1965), 695-708.

[7] Davies, E. B. Semi-Classical States for Non-Self-Adjoint Schrödinger Operators. Communications in Mathematical Physics 200 (1999), 35-41.

[8] Davies, E. B. Wild spectral behaviour of anharmonic oscillators. Bulletin of the London Mathematical Society 32 (2000), 432-438.

[9] Davies, E. B., And KuiJlaArs, A. B. J. Spectral asymptotics of the non-self-adjoint harmonic oscillator. Journal of the London Mathematical Society 70 (2004), 420-426.

[10] Dunford, N., and Schwartz, J. T. Linear Operators, Part 3, Spectral Operators. WileyInterscience, New York-London-Sydney, 1971.

[11] Dunford, N., And Schwartz, J. T. Linear operators, Part 1. John Wiley \& Sons Inc., New York, 1988

[12] Dym, H., And Katsnelson, V. Contributions of Issai Schur to Analysis, Studies in Memory of Issai Schur, vol. 210 of Progr. in Math. Birkhäuser, Boston, MA, 2003. [arXiv:0706.1868].

[13] Feller, W. An Introduction to Probability Theory and Its Applications. New York: Wiley, 1968.

[14] Giertz, M. On the solutions in $L^{2}(-\infty, \infty)$ of $y^{\prime \prime}+(\lambda-q(x)) y=0$ when $q$ is rapidly increasing. Proceedings of the London Mathematical Society 14 (1964), 53-73.

[15] Gohberg, I. C., And Krein, M. G. Introduction to the Theory of Linear Nonselfadjoint Operators in Hilbert Space. AMS, Providence, 1969.

[16] Halmos, P. R., ANd Sunder, V. S. Bounded integral operators on $L^{2}$ spaces, vol. 96 of Ergebnisse der Mathematik und ihrer Grenzgebiete (Results in Mathematics and Related Areas). Springer-Verlag, Berlin, 1978.

[17] Henry, R. Spectral instability of some non-selfadjoint anharmonic oscillators. Comptes Rendus Mathématique. Académie des Sciences. Paris 350 (2012), 1043-1046.

[18] Henry, R. Spectral instability for even non-selfadjoint anharmonic oscillators. Journal of Spectral Theory 4 (2014), 349-364.

[19] Kato, T. Perturbation theory for linear operators. Springer-Verlag, Berlin, 1995.

[20] Markus, A. Introduction to the spectral theory of polynomial operator pencils. American Mathematical Society, 1988.

[21] Milne, W. E. On the maximum absolute value of the derivative of $e^{-x^{2}} p_{n}(x)$. Transactions of the American Mathematical Society 33 (1931), 143-146.

[22] Mityagin, B. The spectrum of a Harmonic Oscillator Operator Perturbed by Point Interactions. arXiv:1407.4153, 2014.

[23] Mityagin, B. The Spectrum of a Harmonic Oscillator Operator Perturbed by Point Interactions. International Journal of Theoretical Physics (2015). DOI 10.1007/s10773-014-2468-z.

[24] Mityagin, B., Siegl, P., And Viola, J. Differential operators admitting various rates of spectral projection growth. arXiv:1309.3751.

[25] Schur, I. Bemerkungen zur Theorie der Beschrankten Bilinearformen mit unendlich vielen Veranderlichten. Journal für die reine und angewandte Mathematik 140 (1911), 1-28.

[26] Shkalikov, A. On the basis property of root vectors of a perturbed self-adjoint operator. Proceedings of the Steklov Institute of Mathematics 269 (2010), 284-298.

[27] Shkalikov, A. Eigenvalue asymptotics of perturbed self-adjoint operators. Methods of Functional Analysis and Topology 18 (2012), 79-89.

[28] Siegl, P., ANd KRejČiřík, D. On the metric operator for the imaginary cubic oscillator. Physical Review D 86 (2012), 121702(R). 
[29] Simon, B. Hamiltonians defined as quadratic forms. Communications in Mathematical Physics 21 (1971), 192-210.

[30] Titchmarsh, E. C. On the asymptotic distribution of eigenvalues. The Quarterly Journal of Mathematics 5 (1954), 228-240.

[31] Titchmarsh, E. C. Eigenfunction expansions associated with second-order differential equations. Part II. Oxford, at the Clarendon Press, 1958.

[32] Wyss, C. Riesz bases for $p$-subordinate perturbations of normal operators. Journal of Functional Analysis 258 (2010), 208-240.

[33] Xu, G. Q., And Yung, S. P. The expansion of a semigroup and a Riesz basis criterion. Journal of Differential Equations 210 (2005), 1-24.

[34] ZALIK, R. A. Inequalities for weighted polynomials. Journal of Approximation Theory 37 (1983), 137-146.

[35] ZwART, H. Riesz basis for strongly continuous groups. Journal of Differential Equations 249 (2010), 2397-2408.

(Boris Mityagin) Department of Mathematics, The Ohio State University, 231 West 18тh Ave, Columbus, OH 43210, USA

E-mail address: mityagin.1@osu.edu

(Petr Siegl) Mathematishes Institut, Universität Bern, Alpeneggstrasse 22, 3012 Bern, Switzerland \& On leave from Nuclear Physics Institute ASCR, 25068 Řž, Czech RePUBLIC

E-mail address: petr.siegl@math.unibe.ch 